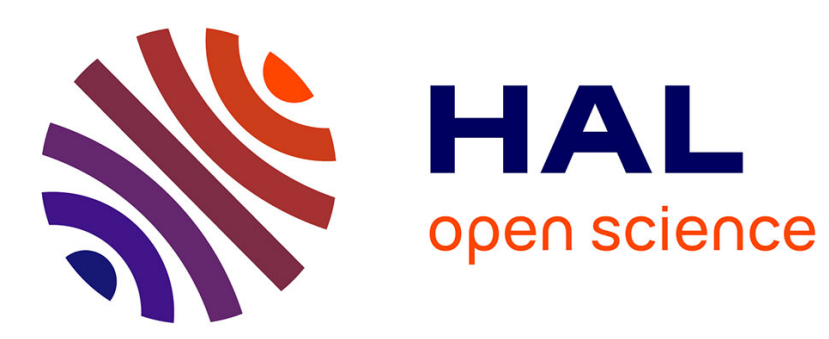

\title{
The Kinematics, Dynamics and Control of a Flying Parallel Robot with Three Quadrotors
}

Damien Six, Sébastien Briot, Abdelhamid Chriette, Philippe Martinet

\section{To cite this version:}

Damien Six, Sébastien Briot, Abdelhamid Chriette, Philippe Martinet. The Kinematics, Dynamics and Control of a Flying Parallel Robot with Three Quadrotors. IEEE Robotics and Automation Letters, 2018, 3 (1), pp.559-566. hal-01634289

\section{HAL Id: hal-01634289 \\ https://hal.science/hal-01634289}

Submitted on 24 Jun 2019

HAL is a multi-disciplinary open access archive for the deposit and dissemination of scientific research documents, whether they are published or not. The documents may come from teaching and research institutions in France or abroad, or from public or private research centers.
L'archive ouverte pluridisciplinaire HAL, est destinée au dépôt et à la diffusion de documents scientifiques de niveau recherche, publiés ou non, émanant des établissements d'enseignement et de recherche français ou étrangers, des laboratoires publics ou privés. 


\title{
The kinematics, dynamics and control of a flying parallel robot with three quadrotors
}

\author{
Damien Six ${ }^{1}$, Sébastien Briot ${ }^{2}$, Abdelhamid Chriette ${ }^{1}$ and Philippe Martinet ${ }^{1}$
}

\begin{abstract}
This paper deals with a novel flying mechanism. Inspired from parallel manipulators, this flying robot is composed of three quadrotors linked by a rigid articulated architecture composed of three legs and a platform. Associating quadrotor co-manipulation and rigid bodies, this new design offers novel possibilities for aerial robotics and manipulation. Previous work leads to the design of a flying parallel robot with two quadrotors. However, this robot did not allow the full control of the six degrees of freedom of its end-effector. With an additional quadrotor, this study seeks to obtain a full control of the platform position and orientation. To prove this property, the kinematic constraints are verified through screw theory. Then, the dynamic model is established and a decoupling property leads to the design of a specific controller for the platform and legs configurations. ADAMS/SIMULINK co-simulations validate the theoretical developments.
\end{abstract}

\section{INTRODUCTION}

The last decade has seen the development of a new generation of aerial robots combining a flying UAV (Unnamed Autonomous Vehicle) with a robotic manipulator. Several options have been explored, from one DoF (Degree of Freedom) manipulator [1] toward more complex serial [2] [3] or parallel [4] [5] robotic devices. The drawbacks of such solutions are a limited payload and autonomy due to the additional embedded motors and also a manipulability impacted by the quadrotor underactuation. To overcome the limits in payload, some studies investigated co-manipulation between several quadrotors in cable-load transportation applications [6]. However, cables cannot apply any pushing forces to the environment, limiting the manipulation area under the drones. Such flying device can also be seen as a cable-driven parallel robot where the actuators have been replaced by quadrotors. Inspired by this analogy, a new type of flying robot can be developed from rigid parallel robots. This new type of flying robots, composed of quadrotors associated to a rigid articulated passive architecture, presents the following advantages [7]

- An enhanced payload, by spreading the efforts over several drones;

- No additional embedded motors, the robot is controlled only by the quadrotors;

- Reconfiguration of the passive architecture potentially allows to perform tasks under and over the drone;

\footnotetext{
${ }^{1}$ Damien Six, Abdelhamid Chriette and Philippe Martinet are with LS2N, UMR CNRS 6004, and Centrale Nantes, Nantes 44321, France Damien.Six@ls2n.fr, Abdelhamid.Chriettedls 2 n.fr,

Phlippe.Martinetals2n.fr

2 Sébastien Briot is with CNRS at LS2N, UMR CNRS 6004 Sebastien.Briotels2n.fr
}

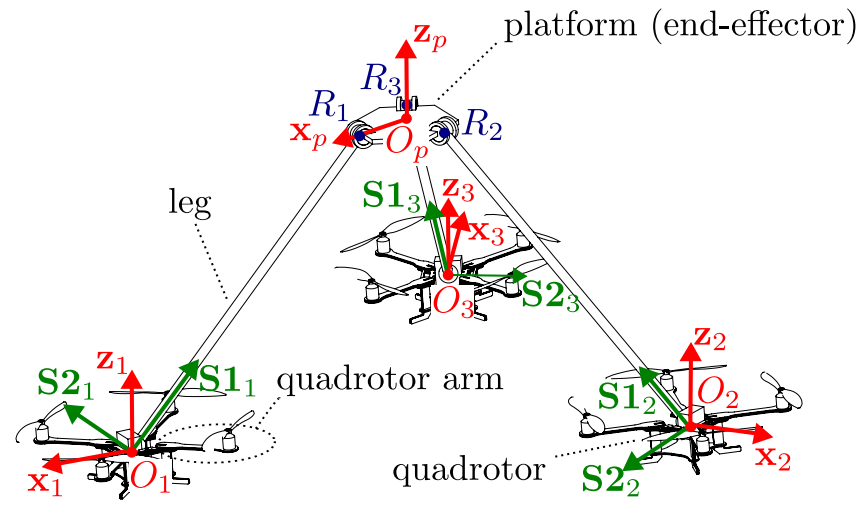

Fig. 1: A flying parallel robot with three quadrotors

- The effector can be placed away from the drone, limiting the perturbations due to rotor wash;

- A large choice of leg topology, issued from the study of parallel robots, can lead to several properties of interest.

A first design with two quadrotors, based on a planar parallel mechanism is described in [7]. The end-effector of this robot was not fully controllable in the six DoFs of space: its evolution was limited to a vertical plane, thus restraining its applicability. This study proposes a new solution based on a spatial architecture with three drones, allowing control of all possible DoFs of the end-effector. The kinematic study in section II shows that this architecture offers full control of the platform position and orientation and it describes its singularity locus. Its dynamic model is developed in section III. Decoupling properties in the dynamic model are exploited to design a cascaded controller in section IV. The simulation results, illustrating the theoretical developments are given in section $\mathrm{V}$.

\section{KINEMATICS}

Figure 1 illustrates the flying parallel robot under study. It consists of a moving platform connected to three quadrotors by means of three identical legs. $\mathcal{F}_{p}\left(O_{p}, \mathbf{x}_{p}, \mathbf{y}_{p}, \mathbf{z}_{p}\right)$ is a frame attached to the moving platform. Its origin $O_{p}$ is the centroid of the platform. $\mathbf{z}_{p}$ axis is normal to the platform top plane. Let $\ell_{A B}$ be the distance between any set of points $A$ and $B$. Three points $R_{i}(i=1,2,3)$ are defined by their coordinates $\overrightarrow{O_{p} R_{i}}=\left[\ell_{O_{p} R_{i}} \cos \gamma_{i}, \ell_{O_{p} R_{i}} \sin \gamma_{i}, 0\right]^{T}$ in frame $\mathcal{F}_{p}$ with $\gamma_{i}=\{0,2 \pi / 3,-2 \pi / 3\} \mathrm{rad}$. A leg $i$ is linked to the platform at point $R_{i}$ by a revolute joint of direction $\mathbf{r}_{i}=\left[-\sin \gamma_{i}, \cos \gamma_{i}, 0\right]^{T}$ expressed in frame $\mathcal{F}_{p}$. Each leg $i$ is attached to a drone $i$ by a spherical joint situated at $O_{i}$ $(i=1,2,3)$ (see Fig. 2). $O_{i}$ is also the center of mass of 


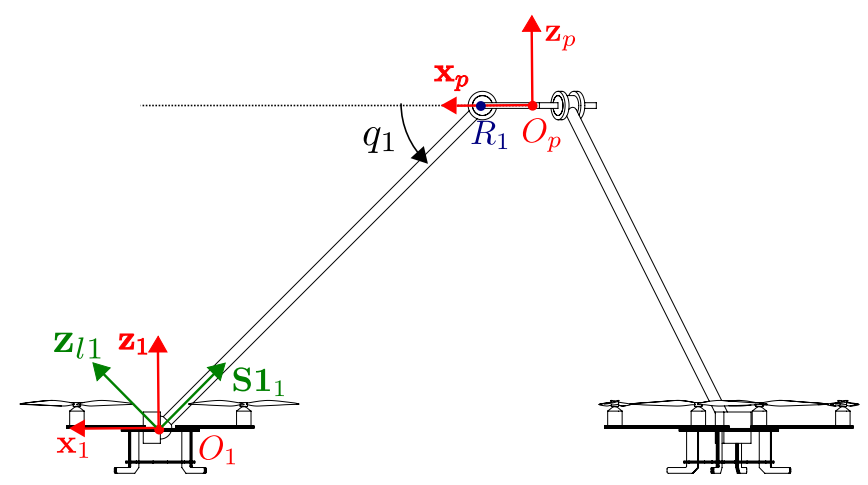

Fig. 2: Side view of the flying parallel robot

drone $i$. The length of leg $i$ is defined by $\ell_{R_{i} O_{i}}$. The actuation of the robot is provided by the three quadrotors.

Attaching the leg at the drone center of mass can be technically challenging, but this configuration is necessary to obtain the full decoupling property on the robot dynamics described in section III. On an experimental device, the centers of the spherical joint may not be exactly situated at the drones center of mass. Then, the centrifugal and Coriolis forces caused by the drones rotation motions will affect the dynamics of the passive architecture and generate additional coupling. With a large distance between the center of mass and the center of rotation, the perturbations induced by this coupling may be higher than the controller stability margin. In [7], the expression of this coupling term is given for a flying robot with two quadrotors and an example illustrates its effect on the tracking precision. Thus, the robustness of the control law against the perturbations induced by this coupling requires to be studied. This point is not addressed in this paper.

\section{A. Parameterization}

Let $\mathcal{F}_{i}\left(O_{i}, \mathbf{x}_{i}, \mathbf{y}_{i}, \mathbf{z}_{i}\right)$ be the frame attached to drone $i(i=$ $1,2,3)$. Its origin $O_{i}$ is at the drone center of mass which is also the center of leg $i$ spherical joint. $\mathbf{x}_{i}$ axis bisects the angle defined by two arms of quadrotor $i . \mathbf{z}_{i}$ axis is aligned with the quadrotor $i$ propellers axes (see Fig. 1).

$\mathbf{q}$ is a vector of the robot generalized coordinates composed of (in the following order)

- $x_{p}, y_{p}, z_{p}, \phi_{p}, \theta_{p}, \psi_{p}$ the position and orientation coordinates of the moving platform frame $\mathcal{F}_{p}$ with respect to the world frame;

- $q_{1}, q_{2}, q_{3}$ the relative angle between the platform plane and leg 1, 2 and 3, respectively (see Fig. 2);

- $\phi_{1}, \theta_{1}, \psi_{1}, \phi_{2}, \theta_{2}, \psi_{2}, \phi_{3}, \theta_{3}, \psi_{3}$ the orientation coordinates of the drone frames $\mathcal{F}_{1}, \mathcal{F}_{2}$ and $\mathcal{F}_{3}$ with respect to the world frame.

Note that in this paper, the rotation sequence of the Bryant angles [8] is chosen such that

$$
\mathbf{v}=\mathbf{R}_{\mathbf{x}}\left(\phi_{i}\right) \mathbf{R}_{\mathbf{y}}\left(\theta_{i}\right) \mathbf{R}_{\mathbf{z}}\left(\psi_{i}\right) \mathbf{v}^{\prime} \quad i=1,2,3 \text { or } p
$$

where $\mathbf{v}$ is a vector expressed in world frame, $\mathbf{v}^{\prime}$ is the same vector expressed in local frame $\mathcal{F}_{i}$ and $\mathbf{R}_{\mathbf{u}}(\alpha)$ is the elemental rotation about axis $\mathbf{u}$ though the angle $\alpha$.

\section{B. Mobility and singularity analyses}

Joining quadrotors with a parallel kinematic architecture combines several challenges. One of them is to be ensured that the platform remains fully controllable. Another challenge is the drones' underactuation: they can only provide an upward thrust force and three independent torques for six DoFs. Classically, for the control of quadrotors, the underactuation is handled through an appropriate controller [9]. In section IV, a cascaded control law is designed. Constraining each drone orientation coordinates by a desired thrust force, this control law allows each drone to apply a force $\mathbf{f}_{i}$ at the associated leg tip (point $O_{i}$ ) in any desired direction. The torques provided by the quadrotors are not transmitted to the parallel architecture through the passive spherical joints. Assuming that such a control law is designed, the kinematic study of the flying robot can be reduced to the study of a passive kinematic architecture, composed of the end-effector and three legs attached to it by the three revolute joints. This architecture is actuated by a force $\mathbf{f}_{i}$ applied at each leg tip in any desired direction.

The kinematic study will first focus on the end-effector mobility and its singularity analysis. Then, the results obtained are extended to the whole passive architecture, including the mobility of each leg.

1) End-effector mobility: Screw theory [10] [11] is suitable for the study of the instantaneous motion of parallel manipulators [12]. A twist and a wrench are screws that represent the instantaneous motion of a rigid body and a system of forces and moments applied on a rigid body, respectively. Screw theory allows to compute the set of constraints, named actuation wrenches, provided by each leg to the platform when the actuators are locked. The rank of the overall wrench system, spanned by the actuation wrenches of all the legs, defines the mobility of the platform. If this system is of dimension six, then the platform if fully controlled. Otherwise, at least one degree of freedom of the platform is not controlled.

For each leg $i(i=1,2,3)$, four unit twists $\boldsymbol{\zeta}_{k i}$ define the instantaneous motions of the leg, one for the revolute joint motion $(k=1)$ and three for the spherical joint motions $(k=2,3,4)$. Any actuation wrench $\boldsymbol{\xi}$ of the leg is reciprocal to all the unit twists denoted $\boldsymbol{\zeta}_{k i}$ characterizing the displacements of the passive joints [12], i.e. $\boldsymbol{\xi}^{T} \boldsymbol{\zeta}_{k i}=0$ for any $k$. This means that the virtual power developed by the wrench $\boldsymbol{\xi}$ along the direction of motion $\boldsymbol{\zeta}_{k i}$ is null. Let us consider one robot leg $i$. To simplify the expressions of the unit twists for this leg, let us define a new frame $\mathcal{F}_{l i}\left(O_{i}, \mathbf{S} \mathbf{1}_{i}, \mathbf{S} \mathbf{2}_{i}, \mathbf{z}_{l i}\right)$ attached to this leg with: the origin $O_{i}$ is at the center of the spherical joint; axis $\mathbf{S} \mathbf{1}_{i}$ pointing toward $R_{i}$ (along the leg); axis $\mathbf{S 2}{ }_{i}$ in the direction of the revolute joint axis (see Figs. 1 and 2). The unit twist defining the motion of the passive revolute joint in frame $\mathcal{F}_{l i}$ at point $O_{i}$ is expressed as [11]

$$
\boldsymbol{\zeta}_{1 i}=\left[\begin{array}{llllll}
0 & 0 & \ell_{R_{i} O_{i}} & 0 & 1 & 0
\end{array}\right]^{T}
$$

while the three unit twists defining the motion of the passive 
spherical joint in frame $\mathcal{F}_{l i}$ at point $O_{i}$ are given by

$$
\begin{aligned}
\boldsymbol{\zeta}_{2 i} & =\left[\begin{array}{llllll}
0 & 0 & 0 & 1 & 0 & 0
\end{array}\right]^{T} \\
\boldsymbol{\zeta}_{3 i} & =\left[\begin{array}{llllll}
0 & 0 & 0 & 0 & 1 & 0
\end{array}\right]^{T} \\
\boldsymbol{\zeta}_{4 i} & =\left[\begin{array}{llllll}
0 & 0 & 0 & 0 & 0 & 1
\end{array}\right]^{T}
\end{aligned}
$$

In these twists, the first three components represent the direction of the translation velocity while the three last components represent the direction of the rotational velocity. For each leg $i$, only two unit actuation wrenches are reciprocal to all twists $\boldsymbol{\zeta}_{k i}$. Their expressions in the frame $\mathcal{F}_{l i}$ at point $O_{i}$ are

$$
\begin{aligned}
& \boldsymbol{\xi}_{1 i}=\left[\begin{array}{llllll}
1 & 0 & 0 & 0 & 0 & 0
\end{array}\right]^{T} \\
& \boldsymbol{\xi}_{2 i}=\left[\begin{array}{llllll}
0 & 1 & 0 & 0 & 0 & 0
\end{array}\right]^{T}
\end{aligned}
$$

in which the first three components represent the direction of the force exerted on the platform and the three last components the direction of the moment. For each leg $i$ $(i=1,2,3), \boldsymbol{\xi}_{1 i}$ and $\boldsymbol{\xi}_{2 i}$ are two forces, exerted on the platform by the leg, applied at $O_{i}$ and directed along $\mathbf{S} \mathbf{1}_{i}$ and $\mathbf{S} \boldsymbol{2}_{i}$, respectively. $\boldsymbol{\xi}_{1 i}$ and $\boldsymbol{\xi}_{2 i}$ can be seen as the two components of the force $\mathbf{f}_{i}$, applied by the drone $i$ on the passive architecture, that are transmitted to the platform. The third component of $\mathbf{f}_{i}$ along $\mathbf{z}_{l i}$ is not transmitted to the endeffector and do not cause any motion of the end-effector. This last component available for each leg $i$ will be used to control the relative orientation between the leg and the platform (see section II-B.3).

Now considering the three robot legs, the overall actuation wrench is given by $\boldsymbol{\Xi}=\left[\boldsymbol{\xi}_{11}, \boldsymbol{\xi}_{21}, \boldsymbol{\xi}_{12}, \boldsymbol{\xi}_{22}, \boldsymbol{\xi}_{13}, \boldsymbol{\xi}_{23}\right]$, with all wrenches expressed in the same frame (e.g. the world frame). This overall wrench system is of dimension 6 , then the endeffector is fully controllable. The next section introduces the singularity analysis, i.e. it studies the singular configurations for which the actuation wrench system locally loses its rank.

2) Singularity analysis of the end effector: There are some tools that define the conditions of degeneracy of a wrench system among which the Grassmann geometry [13] and the Grassmann-Cayley algebra [14] [15]. Regarding our particular case, the Grassmann-Cayley algebra was already used to prove the condition of singularities if the system of wrenches is composed of a triplet of two (independent) forces $\boldsymbol{\xi}_{1 i}$ and $\boldsymbol{\xi}_{2 i}(i=1,2,3), \boldsymbol{\xi}_{1 i}$ and $\boldsymbol{\xi}_{2 i}$ being applied at the same point $O_{i}$ [15]. Let us define four planes

- $\mathcal{P}_{i}(i=1,2,3)$ is the plane passing through point $O_{i}$ and containing the two forces being the resultants of the wrenches $\boldsymbol{\xi}_{1 i}$ and $\boldsymbol{\xi}_{2 i}$ (so it contains the vectors $\mathbf{S} \mathbf{1}_{i}$ and $\left.\mathbf{S}_{2 i}\right)$

- $\mathcal{P}_{4}$ is the plane containing the three points $O_{1}, O_{2}$ and $\mathrm{O}_{3}$.

Conditions of singularities appear if and only if all the four planes $\mathcal{P}_{i}(i=1,2,3,4)$ intersect in (at least) a point, that can be at infinity [15] (see Fig. 3).

Let us illustrate this singularity condition for a case study $\left(\ell_{O_{p} R_{i}}=0.1 \mathrm{~m}\right.$ and $\left.\ell_{R_{i} O_{i}}=0.8 \mathrm{~m}\right)$. Each plane $\mathcal{P}_{i}$ is

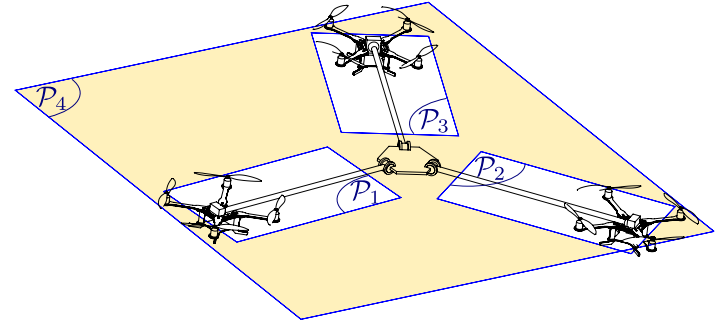

Fig. 3: Example of a singular configuration for the flying parallel robot. $\mathcal{P}_{1}, \mathcal{P}_{2}, \mathcal{P}_{3}$ and $\mathcal{P}_{4}$ are coplanar.

defined by its equation

$$
a_{i} x+b_{i} y+c_{i} z+d_{i}=0
$$

Let $\mathbf{A}$ be the matrix composed of the four plane coefficients

$$
\mathbf{A}=\left[\begin{array}{llll}
a_{1} & b_{1} & c_{1} & d_{1} \\
a_{2} & b_{2} & c_{2} & d_{2} \\
a_{3} & b_{3} & c_{3} & d_{3} \\
a_{4} & b_{4} & c_{4} & d_{4}
\end{array}\right]
$$

The geometric condition of singularity, i.e. all planes intersect in at least a point, is equivalent to $\operatorname{det} \mathbf{A}=\mathbf{0}$. The relative positions of the planes $\mathcal{P}_{1}, \mathcal{P}_{2}, \mathcal{P}_{3}$ and $\mathcal{P}_{4}$ depends only on the mobile robot internal DoFs. Then, the robot singular configurations are not affected by translation or rotation of the whole flying robot in space (i.e. $x_{p}, y_{p}$, $z_{p}, \phi_{p}, \theta_{p}$ and $\psi_{p}$ coordinates). The internal degrees of freedom, i.e. the legs orientation $q_{1}, q_{2}$ and $q_{3}$, are sufficient to study the singularity locus. To avoid auto-collision, each coordinates $q_{i}$ is restricted to range $[-\pi / 2 ; \pi / 2]$. The 2dimensional representations as function of $q_{2}$ and $q_{3}$ of the singularity locus for $q_{1}=0,-\pi / 6,-\pi / 3$ and $-\pi / 2$ are shown in Fig. 4. The singularity locus is obtained through the numerical computation of $|\operatorname{det} \mathbf{A}|$ for which we retain the values "close to zero". For this numerical analysis, a threshold is set at $5 \mathrm{e}-4$. The results for positive values of $q_{1}$ can be obtained by a central symmetry in the origin of the figures. From those figures, we can see that a workspace with each $q_{i}$ restricted to range $[-\pi / 2 ; 0]$ is singularity free. However, a change of configuration with a platform under the quadrotors (all $q_{i}>0$ ) will require the crossing of a singularity. Still, the effective workspace of the robot could be extended using singularity crossing techniques already applied on standard parallel robots [16].

3) Singularity analysis of the passive architecture (endeffector and legs): Only two components of each force provided by the drones are transmitted to the platform (see section II-B.1). Then, one component by leg remains available to control an additional degree of freedom: one pure force along $\mathbf{z}_{l i}$. This remaining inputs on leg $i$ will allow the control of the leg configuration $q_{i}$ (see Fig. 2). In section II-B.2, the singularity conditions have been studied for the end-effector alone. In this section, by expressing the Jacobian matrix for the whole passive structure (end-effector and legs), it will be proven that including those additional coordinates do not change the conditions of singularity. Let 


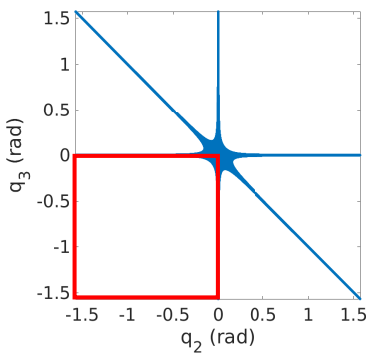

(a) $q_{1}=0 \mathrm{rad}$

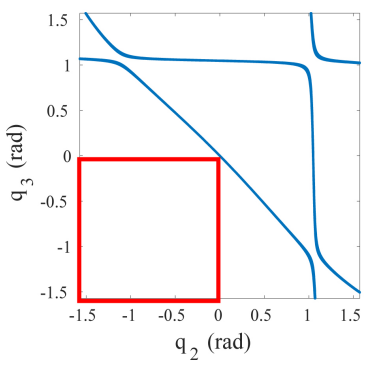

(c) $q_{1}=-\pi / 3 \mathrm{rad}$
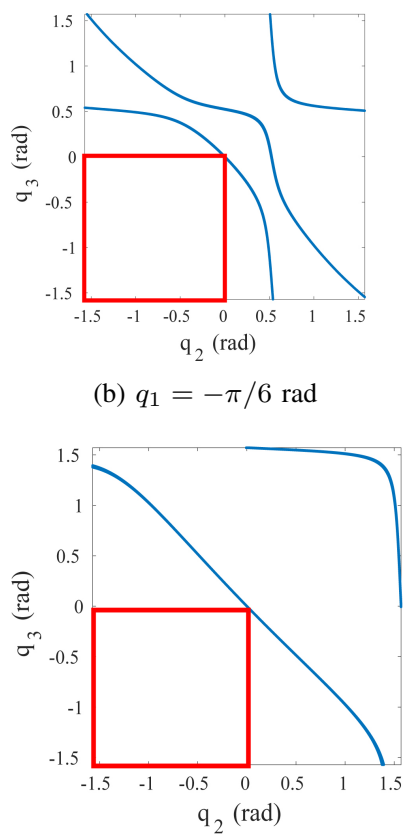

(b) $q_{1}=-\pi / 6 \mathrm{rad}$

(d) $q_{1}=-\pi / 2 \mathrm{rad}$

Fig. 4: Singularity locus $(|\operatorname{det} \mathbf{A}|<5 \mathrm{e}-4$ ) for the passive architecture with $\ell_{O_{p} R_{i}}=0.1 \mathrm{~m}$ and $\ell_{R_{i} O_{i}}=0.8 \mathrm{~m}$. The red square areas represent a singularity free workspace.

$\mathbf{q}_{p}=\left[x_{p}, y_{p}, z_{p}, \phi_{p}, \theta_{p}, \psi_{p}, q_{1}, q_{2}, q_{3}\right]^{T}$ be the generalized coordinates of the passive architecture only. Let $\mathbf{v}_{i}(i=$ $1,2,3)$ be the velocity of the point $O_{i}$ (leg tip) expressed in world frame and $\mathbf{J}$ be the Jacobian matrix such that

$$
\mathbf{v}=\mathbf{J} \dot{\mathbf{q}}_{p}
$$

with $\mathbf{v}=\left[\mathbf{v}_{1}^{T}, \mathbf{v}_{2}^{T}, \mathbf{v}_{3}^{T}\right]^{T}$. A singularity of this $(9 \times 9)$ matrix corresponds to a robot configuration where an infinitesimal motion of the passive architecture coordinates $\mathbf{q}_{p}$ is possible even if the leg tips are fixed.

To study the singularities of this matrix, its is convenient to change the reference frame. For each $i=(1,2,3)$, let $v_{1 i}$ (respectively $v_{2 i}$ and $v_{3 i}$ ) be the coordinate of $\mathbf{v}_{i}$ along $\mathbf{S} \mathbf{1}_{i}$ (respectively $\mathbf{S} \mathbf{2}_{i}$ and $\mathbf{z}_{l i}$ ). The vector $\mathbf{v}_{l}$ is defined by

$$
\mathbf{v}_{l}=\left[\begin{array}{lllllllll}
v_{11} & v_{12} & v_{12} & v_{21} & v_{22} & v_{23} & v_{31} & v_{32} & v_{33}
\end{array}\right]^{T}
$$

Let $\mathbf{J}_{l}$ be a Jacobian matrix such that

$$
\mathbf{v}_{l}=\mathbf{J}_{l} \dot{\mathbf{q}}_{p}
$$

$\mathbf{J}$ and $\mathbf{J}_{l}$ are related through a full rank matrix defined by the rotations from the world frame to the local frames $\mathcal{F}_{l i}$ and the coordinate ordering defined in (11). Skipping the mathematical calculations, $\mathbf{J}_{l}$ takes the form

$$
\mathbf{J}_{l}=\left[\begin{array}{cccc}
\mathbf{J}_{r} & & \mathbf{0}_{6 \times 3} & \\
& \ell_{R_{1} O_{1}} & & 0 \\
\star & 0 & \ell_{R_{2} O_{2}} & 0 \\
& 0 & 0 & \ell_{R_{3} O_{3}}
\end{array}\right]
$$

with $\mathbf{J}_{r}$ linking the velocity of the leg tips along $\mathbf{S} \mathbf{1}_{i}$ and $\mathbf{S 2} \mathbf{z}_{i}$ only $(i=1,2,3)$ to the derivative of the end-effector coordinates, i.e.

$$
\left[\begin{array}{llllll}
v_{11} & v_{12} & v_{13} & v_{21} & v_{22} & v_{23}
\end{array}\right]^{T}=\mathbf{J}_{r} \dot{\mathbf{q}}_{e}
$$

$\mathbf{q}_{e}=\left[x_{p}, y_{p}, z_{p}, \phi_{p}, \theta_{p}, \psi_{p}\right]^{T}$ is the vector of the endeffector coordinates. $\mathbf{J}_{r}$ is thus the inverse Jacobian matrix of the passive architecture in the case where the drones are able to exert motions (but also forces) along $\mathbf{S} \mathbf{1}_{i}$ and $\mathbf{S 2}_{i}(i=1,2,3)$ only. It is known from [17] that the rank of this Jacobian matrix is equal to the rank of the overall wrench system. This overall wrench, composed of units forces applied on the end-effector by the legs along $\mathbf{S} \mathbf{1}_{i}$ and $\mathbf{S 2}{ }_{i}$, is the one studied in section II-B.2. If the endeffector is not in a singular configuration, shown in section II-B.2, then $\mathbf{J}_{r}$ is of full rank.

By (13), the rank of the matrix $\mathbf{J}_{l}$ is equal to the rank of $\mathbf{J}_{r}$ plus 3, both matrices have the same conditions of rank deficiency. Then, the singularity locus of the whole passive architecture is equivalent to the singularity locus of the endeffector given in section II-B.2.

\section{DYNAMICS}

The major difficulty in controlling quadrotor is underactuation. A quadrotor can only produce three independent torques and an upward thrust force to control its six DoF in space [9]. In a single quadrotor dynamic model, the attitude dynamics is independent from its position dynamics and is controlled only through torques, while the translational dynamics only depends on the thrust force and the drone orientation. This property is exploited to design cascaded controllers for quadrotors. Because the spherical joint centers are situated at the drones center of mass, the attitude dynamics of the quadrotors are not linked to the passive architecture dynamics, allowing to find the same kind of decoupling. In this section, the dynamic equations of the passive architecture and the quadrotor attitude are given. The following assumptions are made. On each quadrotor, four control inputs are available, one thrust input $u_{1 i}$ and three torques $\boldsymbol{\tau}_{i}=\left[u_{2 i}, u_{3 i}, u_{4 i}\right]^{T}$. The rotor dynamics is considered fast before the body dynamics and is neglected in the scope of this study. Disturbances from aerodynamic effects are also neglected in the model. In this section, the dynamic equations of the passive architecture alone will first be established. Then, the Newton-Euler equations, applied at the drones center of gravity, will give two set of dynamic equations: one linking the drones attitude dynamics to the input torques and one linking the passive architecture dynamics to the drone thrust forces.

\section{A. Passive architecture dynamics}

From the quadrotors, only one force $\mathbf{f}_{i}$ per quadrotor $i$ $(i=1,2,3)$ is transmitted to the passive architecture through the passive spherical joints at each leg tip. Let $L\left(\mathbf{q}_{p}, \dot{\mathbf{q}}_{p}\right)$ the Lagrangian of the passive architecture. The Lagrangian 
equation of the architecture with three forces $\left(\mathbf{f}_{1}, \mathbf{f}_{2}, \mathbf{f}_{3}\right)$ applied on the leg tips are

$$
\frac{\mathrm{d}}{\mathrm{dt}}\left(\frac{\partial L}{\partial \dot{\mathbf{q}}_{p}}\right)^{T}-\left(\frac{\partial L}{\partial \mathbf{q}_{p}}\right)^{T}=\mathbf{J}^{T}\left[\begin{array}{l}
\mathbf{f}_{1} \\
\mathbf{f}_{2} \\
\mathbf{f}_{3}
\end{array}\right]
$$

with $\mathbf{J}$ a Jacobian matrix defined in (10) that remains full rank out of singularities as described in section II-B.2.

The robot is now considered to remain away from singularities. Equation (15) can be written as [18]

$$
\mathbf{M}_{p} \ddot{\mathbf{q}}_{p}+\mathbf{c}_{p}=\mathbf{J}^{T}\left[\begin{array}{l}
\mathbf{f}_{1} \\
\mathbf{f}_{2} \\
\mathbf{f}_{3}
\end{array}\right]
$$

where $\mathbf{M}_{p}$ is the $(9 \times 9)$ generalized inertia matrix and $\mathbf{c}_{p}$ the 9-dimensional vector of Coriolis, centrifugal and gravitational effects.

\section{B. Quadrotor dynamics}

Only reaction forces $\left(-\mathbf{f}_{1},-\mathbf{f}_{2}\right.$ and $\left.-\mathbf{f}_{3}\right)$ are transmitted to the drones from the passive architecture through the spherical joints. The Newton-Euler laws applied at the drone $i$ center of mass give

$$
\begin{aligned}
m \dot{\mathbf{v}}_{i} & =m \mathbf{g}-\mathbf{f}_{i}+\mathbf{t}_{i} \\
\boldsymbol{\tau}_{i} & =\mathbf{I}_{i} \dot{\boldsymbol{\omega}}_{i}+\boldsymbol{\omega}_{i} \times \mathbf{I}_{i} \boldsymbol{\omega}_{i}
\end{aligned}
$$

where

- $m$ the mass of one drone ${ }^{1}$;

- $\mathbf{t}_{i}$ the thrust force of drone $i$;

- $\mathbf{g}$ the gravity vector

- $\boldsymbol{\tau}_{i}$ is the torque vector of quarotor $i$ in local frame;

- $\boldsymbol{\omega}_{i}$ is the angular velocity of quarotor $i$ in local frame ;

- $\mathbf{I}_{i}$ is the inertia matrix of quadrotor $i$.

The angular velocity of a quadrotor $i$ is related to the derivative of its coordinates through the relation

$$
\boldsymbol{\omega}_{i}=\mathbf{T}_{i} \dot{\boldsymbol{\eta}}_{i}
$$

with $\boldsymbol{\eta}_{i}=\left[\phi_{i}, \theta_{i}, \psi_{i}\right]^{T}$ and

$$
\mathbf{T}_{i}=\left[\begin{array}{ccc}
\cos \theta_{i} \cos \psi_{i} & \sin \psi_{i} & 0 \\
-\cos \theta_{i} \sin \psi_{i} & \cos \psi_{i} & 0 \\
\sin \theta_{i} & 0 & 1
\end{array}\right]
$$

This matrix depends on the choice in the orientation representation which is defined in (1) for this study. Introducing (19) in (18) gives

$$
\boldsymbol{\tau}_{i}=\mathbf{I}_{i}\left(\mathbf{T}_{i} \ddot{\boldsymbol{\eta}}_{i}+\dot{\mathbf{T}}_{i} \dot{\boldsymbol{\eta}}_{i}\right)+\mathbf{T}_{i} \dot{\boldsymbol{\eta}}_{i} \times \mathbf{I}_{i} \mathbf{T}_{i} \dot{\boldsymbol{\eta}}_{i}
$$

Equation (21) is the attitude coordinate dynamic model for each drone as function of its input torques. This equation is independent from the thrust force and the passive architecture dynamics.

\footnotetext{
${ }^{1}$ Masses of the three drones are considered equal to simplify notations, but the result can easily be extended to drones with various masses.
}

\section{Whole robot dynamics}

Equations (17) and (16) link the translational dynamics of the drones and the passive architecture dynamics through the reaction forces. Introducing (17) and the time derivative of (10) in (16) gives, skipping the mathematical calculations,

$$
\mathbf{M} \ddot{\mathbf{q}}_{p}+\mathbf{c}=\mathbf{J}^{T} \mathbf{t}
$$

with

$$
\begin{aligned}
& \text { - } \mathbf{t}=\left[\mathbf{t}_{1}^{T}, \mathbf{t}_{2}^{T}, \mathbf{t}_{3}^{T}\right]^{T} \\
& \text { - } \mathbf{M}=\mathbf{M}_{p}+m \mathbf{J}^{T} \mathbf{J} \\
& \text { - } \mathbf{c}=\mathbf{c}_{p}+m \mathbf{J}^{T} \dot{\mathbf{J}} \dot{\mathbf{q}}_{p}-m \mathbf{J}^{T}\left[\mathbf{g}^{T}, \mathbf{g}^{T}, \mathbf{g}^{T}\right]^{T}
\end{aligned}
$$

Another way to compute directly this expression is to consider an equivalent passive architecture that has an additional mass corresponding to one quadrotor mass at each leg tip with only the thrust force of each drone applied to the leg tip. Equation (22) gives the dynamic equations of the passive structure coordinates $\mathbf{q}_{p}$ as function of the drones thrust forces only. The thrust force of a drone $i$ in world frame is related to its thrust input $u_{1 i}$ by

$$
\mathbf{t}_{i}=\mathbf{R}_{i}\left[\begin{array}{lll}
0 & 0 & u_{1 i}
\end{array}\right]^{T}
$$

with $\mathbf{R}_{i}=\mathbf{R}_{\mathbf{x}}\left(\phi_{i}\right) \mathbf{R}_{\mathbf{y}}\left(\theta_{i}\right) \mathbf{R}_{\mathbf{z}}\left(\psi_{i}\right)$ the rotation matrix of the local frame $\mathcal{F}_{i}$.

Equations (21) and (22) are the two set of equations expressing the dynamics of the whole robot. They show a decoupling between the passive structure dynamics and the drones attitude dynamics. However, the decoupling is not complete as the thrust forces $\mathbf{t}$ in the passive structure dynamic equations (22) depends on the thrust inputs $u_{1 i}$ but also on the orientation coordinates of the drones (23). Section IV-A shows how this coupling can be handled by binding the desired drone coordinates to the desired thrust forces.

\section{CONTROL}

The main challenge to control the full architecture is linked to the quadrotors underactuation. However, imposing a condition on the quadrotors orientation coordinates allows the definition of a virtual input that decouples the dynamics of the passive architecture from the drone attitude coordinates. Based on this property, a two steps controller is established. The global scheme of this controller is given in Fig. 5.

\section{A. Decoupling for control of the passive architecture}

The design of the passive architecture control law is based on feedback linearisation of the robot dynamics. Such controller is well known for the control of robot manipulators [18] but has also been used for the control quadrotors [19]. From the robot dynamic model (22), let us define a virtual control input vector $\nu_{1}$

$$
\boldsymbol{\nu}_{1}=\mathbf{M}^{-1}\left(\mathbf{J}^{T} \mathbf{t}-\mathbf{c}\right)
$$

If such control vector exists, then introducing (24) in (22) gives the double integrator

$$
\ddot{\mathbf{q}}_{p}=\boldsymbol{\nu}_{1}
$$




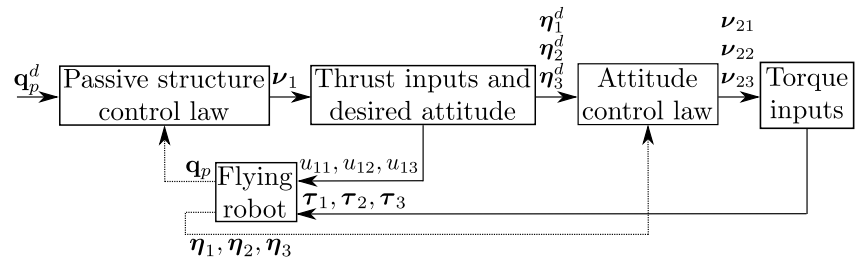

Fig. 5: General controller scheme

From (24), the desired thrust forces $\mathbf{t}^{d}=\left[\mathbf{t}_{1}^{d^{T}}, \mathbf{t}_{2}^{d^{T}}, \mathbf{t}_{3}^{d^{T}}\right]^{T}$ in world frame to obtain the virtual control input $\nu_{1}$ are given by

$$
\mathbf{t}^{d}=\mathbf{J}^{-T}\left(\mathbf{M} \nu_{1}+\mathbf{c}\right)
$$

From (23), the thrust force provided by each drone depends on its thrust input and its orientation coordinates

$$
\mathbf{t}_{i}=\left[\begin{array}{c}
\sin \theta_{i} \\
-\sin \phi_{i} \cos \theta_{i} \\
\cos \phi_{i} \cos \theta_{i}
\end{array}\right] u_{1 i}
$$

The desired thrust $\mathbf{t}^{d}$ in (26) force may only be obtained for a given orientation of each quadrotor. From (27), this desired orientation for a quadrotor $i$ is given by the following angles

$$
\begin{aligned}
\phi_{i}^{d} & =-\operatorname{atan} 2\left(t_{y i}^{d}, t_{z i}^{d}\right) \\
\theta_{i}^{d} & =\operatorname{asin}\left(\frac{t_{x i}^{d}}{\left\|\mathbf{t}_{i}^{d}\right\|_{2}}\right)
\end{aligned}
$$

with $\mathbf{t}_{i}^{d}=\left[t_{x i}^{d}, t_{y i}^{d}, t_{z i}^{d}\right]^{T} . t_{z i}>0$ is assumed in all flight configurations (which is the case as long as the dynamic forces and the passive architecture reaction forces along $\mathbf{z}$ axis remain lower than the drone weight). The thrust input $u_{1 i}$ is defined by the projection of the desired thrust $\mathbf{t}_{i}^{d}$ in world frame onto the local $\mathbf{z}_{i}$ axis of the drone

$$
u_{1 i}=\sin \theta_{i} t_{x i}^{d}-\cos \theta_{i} \sin \phi_{i} t_{y i}^{d}+\cos \phi_{i} \cos \theta_{i} t_{z i}^{d}
$$

Suppose $\theta_{i}=\theta_{i}^{d}$ and $\phi_{i}=\phi_{i}^{d}$, then introducing (28) and (29) in (30) gives, skipping the mathematical calculations

$$
u_{1 i}=\left\|\mathbf{t}_{\mathbf{i}}{ }^{d}\right\|_{2}
$$

And introducing (28), (29) and (31) in (23)

$$
\mathbf{t}_{i}=\mathbf{R}_{i}\left[\begin{array}{lll}
0 & 0 & u_{1 i}
\end{array}\right]^{T}=\mathbf{t}_{\mathbf{i}}{ }^{d} \quad i=1,2,3
$$

Equation (32) proves that the desired thrust force $\mathbf{t}^{d}$ as well as the virtual control input $\boldsymbol{\nu}_{1}$ (24) can be obtained as long as the desired attitudes angles defined by (28) and (29) are respected with thrust inputs given by (30) on each quadrotor.

\section{B. Passive architecture control law}

Suppose a trajectory $\mathbf{q}_{p}^{d}$ defined for the coordinates of the passive architecture. Let us design a control law for the virtual input $\boldsymbol{\nu}_{1}$. As $\boldsymbol{\nu}_{1}$ is the control input of a double integrator (25), any control law that stabilizes a double integrator can be designed. A PD (Proportional-Derivative) controller is then designed

$$
\boldsymbol{\nu}_{1}=\ddot{\mathbf{q}}_{p}^{d}-K_{p}\left(\mathbf{q}_{p}-\mathbf{q}_{p}^{d}\right)-K_{d}\left(\dot{\mathbf{q}}_{p}-\dot{\mathbf{q}}_{p}^{d}\right)
$$

with $K_{p}, K_{d}$ strict positive gains to ensure the closed-loop stability.

\section{Quadrotors attitude control law}

The control of the attitude coordinates of the quadrotors is based on Sliding Mode Control (SMC). SMC have already been applied for the control of a single quadrotor attitude in [20]. The reference trajectories of the drone $i(i=1,2,3)$ attitude coordinates $\phi_{i}^{d}$ and $\theta_{i}^{d}$ are defined by (28) and (29). The reference trajectories for the last coordinate on each drone $\psi_{i}^{d}$ remains free to be defined by the user. It can be exploited to avoid the collision between the drone arms and the legs. Those three reference trajectories for each drone forms the desired attitude vector $\boldsymbol{\eta}_{i}^{d}=\left[\phi_{i}^{d}, \theta_{i}^{d}, \psi_{i}^{d}\right]^{T}$. From (21), three auxiliary inputs (one for each drone) are defined

$$
\boldsymbol{\nu}_{2 i}=\mathbf{T}_{i}^{-1} \mathbf{I}_{i}^{-1}\left(\boldsymbol{\tau}_{i}-\mathbf{T}_{i} \dot{\boldsymbol{\eta}}_{i} \times \mathbf{I}_{i} \mathbf{T}_{i} \dot{\boldsymbol{\eta}}_{i}\right)-\mathbf{T}_{i}^{-1} \dot{\mathbf{T}}_{i} \dot{\boldsymbol{\eta}}_{i}
$$

Introducing (34) in (21) gives

$$
\boldsymbol{\nu}_{2 i}=\ddot{\boldsymbol{\eta}}_{i}
$$

Each auxiliary input $\boldsymbol{\nu}_{2 i}$ acts as a double integrator on the drone attitude coordinates $\boldsymbol{\eta}_{i}$. A fast convergence is required on the attitude closed-loop system as the desired angles are necessary to obtain the convergence on the passive architecture coordinates (see section IV-A). To ensure also robustness against model errors, a SMC is defined. Let $\sigma_{i}$ be the sliding variable for each quadrotor

$$
\boldsymbol{\sigma}_{i}=\left(\dot{\boldsymbol{\eta}}_{i}-\dot{\boldsymbol{\eta}}_{i}^{d}\right)+\lambda\left(\boldsymbol{\eta}_{i}-\boldsymbol{\eta}_{i}^{d}\right)
$$

with $\lambda>0$. The sliding mode control law is defined by

$$
\boldsymbol{\nu}_{2 i}=\ddot{\boldsymbol{\eta}}_{i}^{d}-\lambda\left(\dot{\boldsymbol{\eta}}_{i}-\dot{\boldsymbol{\eta}}_{i}^{d}\right)-K_{s} \operatorname{sign}\left(\boldsymbol{\sigma}_{i}\right)
$$

where $K_{s}>0$ and the sign(.) function of a $n$-dimensional vector $\sigma$ is the $n$-dimensional vector $\mathbf{s}$ defined by

$$
\mathbf{s}^{j}=\left\{\begin{array}{rll}
1 & \text { if } & \boldsymbol{\sigma}^{j}>0 \\
0 & \text { if } & \boldsymbol{\sigma}^{j}=0 \\
-1 & \text { if } & \boldsymbol{\sigma}^{j}<0
\end{array}\right.
$$

where $\mathbf{s}^{j}$ is the $i^{\text {th }}$ element of a vector $\mathbf{s}$. Consider now the Lyapunov function candidate

$$
V_{i}=\boldsymbol{\sigma}_{i}^{T} \boldsymbol{\sigma}_{i}
$$

Taking the first time derivative of (39) by considering (35), (36) and (37), we have

$$
\dot{V}_{i}=-2 K_{s} \boldsymbol{\sigma}_{i}^{T} \operatorname{sign}\left(\boldsymbol{\sigma}_{i}\right)
$$

$\dot{V}_{i}$ is negative definite for $K_{s}>0$ therefore the stability and convergence of $\sigma_{i}$ toward zero is ensured. The stability is also ensured with any bounded disturbances by increasing the gain $K_{s}$. The sliding mode control law ensures that the manifold $\boldsymbol{\sigma}_{i}=\mathbf{0}$ is reached in finite time. Once on the manifold, the convergence of the attitude coordinates $\boldsymbol{\eta}_{i}$ towards the desired one $\boldsymbol{\eta}_{i}^{d}$ is guaranteed by (36) with $\lambda>0$.

One drawback of the sliding mode control is that it generates chattering on the control inputs. To reduce this chattering effect, the sign(.) function is replaced by the sat(.) 


\begin{tabular}{lc} 
Parameter & Value \\
\hline Platform mass $(\mathrm{kg})$ & {$\left[\begin{array}{ccc}0.03 & 0 & 0 \\
0 & 0.03 & 0 \\
0 & 0 & 0.05\end{array}\right]$} \\
$\begin{array}{l}\text { Platform inertia matrix }\left(\mathrm{kg} . \mathrm{m}^{2}\right) \\
\text { in frame } \mathcal{F}_{p}\end{array}$ & {$\left[\begin{array}{ccc}0.00001 & 0.6 & 0 \\
0 & 0.01 & 0 \\
0 & 0 & 0.01\end{array}\right]$} \\
\hline Leg mass $(\mathrm{kg})$ & $\begin{array}{l}\text { Leg inertia matrix }\left(\mathrm{kg} . \mathrm{m}^{2}\right) \\
\text { in frame } \mathcal{F}_{l i}\end{array}$ \\
\hline Drone mass $(\mathrm{kg})$ & $\left.\begin{array}{ccc}0.015 & 0 & 0 \\
0 & 0.015 & 0 \\
0 & 0 & 0.03\end{array}\right]$
\end{tabular}

TABLE I: Mass and inertia parameters for simulation

\begin{tabular}{c|c||c|c}
$\begin{array}{c}\text { Passive architecture } \\
\text { coordinates }\end{array}$ & $\begin{array}{c}\text { Error mean } \\
\text { (rad or m) }\end{array}$ & Drone coordinates & $\begin{array}{c}\text { Error mean } \\
\text { (rad) }\end{array}$ \\
\hline$x$ & $3.8 \mathrm{e}-3$ & $\psi_{1}$ & $4.7 \mathrm{e}-4$ \\
$y$ & $7.2 \mathrm{e}-4$ & $\theta_{1}$ & $1.3 \mathrm{e}-3$ \\
$z$ & $2.7 \mathrm{e}-3$ & $\phi_{1}$ & $1.4 \mathrm{e}-3$ \\
$\psi$ & $1.7 \mathrm{e}-3$ & $\psi_{2}$ & $4.5 \mathrm{e}-4$ \\
$\theta$ & $4.4 \mathrm{e}-3$ & $\theta_{2}$ & $7.3 \mathrm{e}-4$ \\
$\phi$ & $5.0 \mathrm{e}-3$ & $\phi_{2}$ & $7.8 \mathrm{e}-4$ \\
$q_{1}$ & $7.3 \mathrm{e}-3$ & $\psi_{3}$ & $4.5 \mathrm{e}-4$ \\
$q_{2}$ & $5.6 \mathrm{e}-3$ & $\theta_{3}$ & $5.2 \mathrm{e}-4$ \\
$q_{3}$ & $6.5 \mathrm{e}-3$ & $\phi_{3}$ & $4.2 \mathrm{e}-4$
\end{tabular}

TABLE II: Mean of the absolute value of the filtered error on each coordinate obtained in simulation

function. The sat(.) function of a $n$-dimensional vector $\sigma$ is the $n$-dimensional vector $\mathbf{s}$ defined by

$$
\mathbf{s}^{j}=\left\{\begin{array}{rll}
1 & \text { if } & \boldsymbol{\sigma}^{j}>\epsilon \\
\boldsymbol{\sigma}^{j} / \epsilon & \text { if } & \left|\boldsymbol{\sigma}^{j}\right| \leq \epsilon \\
-1 & \text { if } & \boldsymbol{\sigma}^{j}<-\epsilon
\end{array}\right.
$$

$\epsilon$ defines a thin boundary layer neighboring the sliding surface. The stability beyond this boundary layer is guaranteed, and the system remains in this boundary once entering it.

\section{Overall control system}

The overall control system consists in the inner attitude and the outer passive architecture control loops, forming the structure as shown in Fig. 5. The two control systems determine the twelve input variables $u_{1 i}, u_{2 i}, u_{3 i}$ and $u_{4 i}$ for $i=(1,2,3)$. The feedback linearization for the passive architecture control law takes into account the dynamics nonlinear behavior of the robot. The sliding mode control for the attitude control law ensures a fast convergence of the attitude coordinates and robustness against disturbances.

\section{SIMULATION}

A simulator has been developed using co-simulation between the softwares ADAMS and MATLAB/SIMULINK. A discrete sample time of communication is set at $1 \mathrm{kHz}$ between the two softwares. The geometrical parameters for the simulation are $\ell_{O_{p} R_{i}}=0.1 \mathrm{~m}$ and $\ell_{R_{i} O_{i}}=0.8 \mathrm{~m}$. The dynamics parameters are given in Table I. To test the robustness of the controller against model errors, an aerodynamic perturbation is implemented. On each drone a

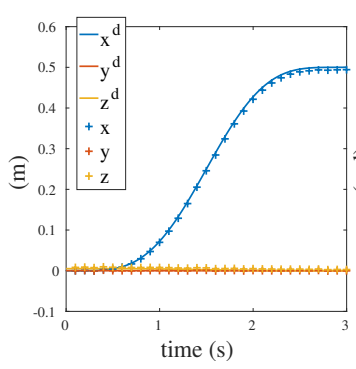

(a) Platform translation

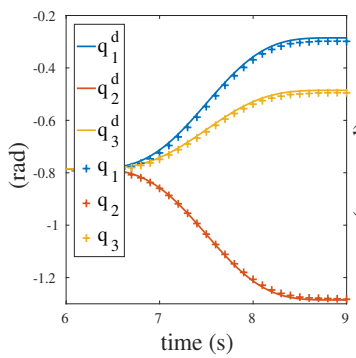

(c) Legs motion

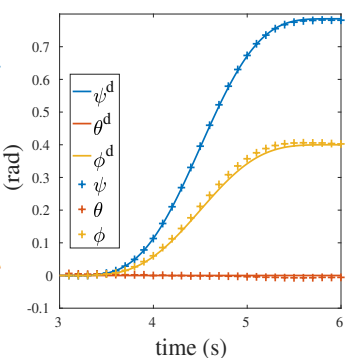

(b) Platform rotation

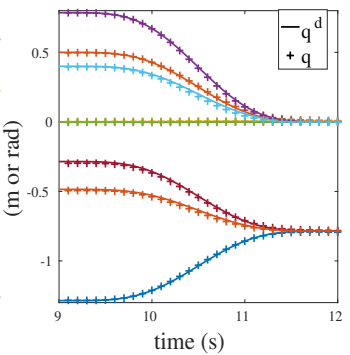

(d) Global motion
Fig. 6: Desired trajectory and filtered tracked trajectory in simulation for successive motions of the passive architecture

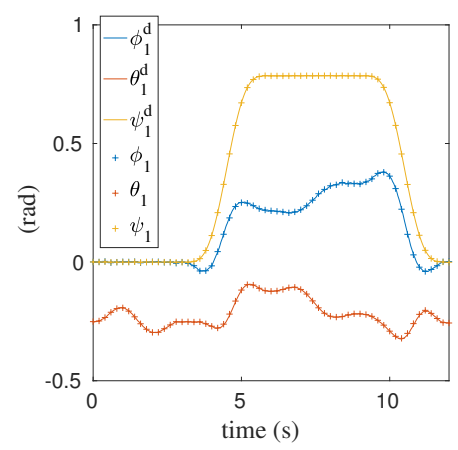

Fig. 7: Desired trajectory and filtered tracked trajectory for one drone attitude in simulation

perturbation force is defined in local frame by

$$
\mathbf{a}_{i}=-\mathbf{A} \mathbf{v}_{i}
$$

where $\mathbf{A}$ is a diagonal friction matrix. The friction coefficients are the same for each direction $\left(1 \mathrm{~kg} . \mathrm{s}^{-1}\right)$. Also, a white noise of amplitude $10^{-2}$ ( $\mathrm{m}$ or rad) is added to the robot state measurements.

The coefficients of the control laws defined in section IV are $K_{p}=4, K_{d}=4, \lambda=15, K_{s}=10, \epsilon=1$. Successive trajectories, defined by nine-degrees polynomials have been designed to test the several degrees-of-freedom of the robot (translation, rotation of the platform and legs orientation). Those trajectories and the tracking obtained are show in Fig. 6. Fig. 7 shows the drone 1 desired attitude coordinates and the tracking of those coordinates for the whole simulation. The controller robustly completes the mission under disturbances and sensor noise. Table II gives the mean of absolute value of the filtered error for each 


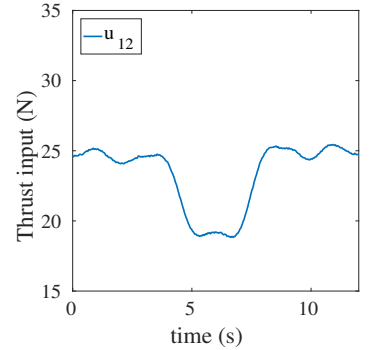

(a) Thrust force

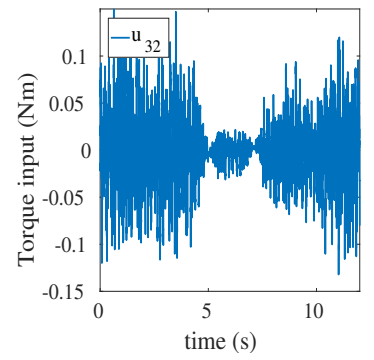

(c) Torque along $\mathbf{y}_{2}$

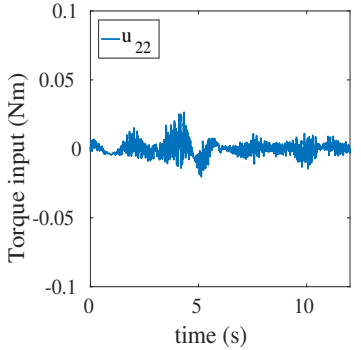

(b) Torque along $\mathbf{x}_{2}$

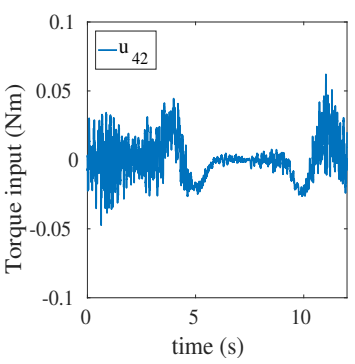

(d) Torque along $\mathbf{z}_{2}$
Fig. 8: Torque and forces input for drone 2 in simulation

coordinate for the whole simulation.

Figure 8 shows the thrust force and torque inputs on drone 2 during the whole simulation. The chattering effect is induced on torques by the high level of noise on the sensors. The parameter $\epsilon$ acts as a trade-off between stability and level of chattering effect. A video of the simulation is available in the attached media content. In this video content, an example of a task that can be performed by such device is also illustrated. For this task, the robot has to insert a tool into a hole and provide a rotation motion about the vertical axis while maintaining the effector in the hole. This task illustrates the ability of the system to perform a rotation of the platform along a direction without any coupling along the other coordinates.

\section{CONCLUSION}

In this paper, a new type of flying robot was proposed. The robot is composed of three quadrotors linked by a rigid articulated passive architecture. This new flying robot offers the ability to control a platform position and orientation in space, with a high potential to perform aerial operations from this platform. The study of the dynamic model showed decoupling properties. Those properties have been exploited to design a cascaded controller adapted for this new flying robot. Simulations showed controller performance and robustness against noisy pose estimation and disturbances. With a white noise of amplitude $10^{-2}$ (m or rad) on the robot state measurements, the controller showed a mean error lower than $7.5 \mathrm{e}-3(\mathrm{~m}$ or $\mathrm{rad})$ on the passive architecture coordinates and lower than $1.5 \mathrm{e}-3 \mathrm{rad}$ on the orientation coordinates.

Future work includes exploring the application of reconfiguration techniques known for parallel robots on the flying robot to increase its workspace. Thus, more possibilities will be offered for the relative position of the drones and the platform. Physical interactions with the environment can also be explored, leading to applications in aerial manipulation. Finally, the extension of the theoretical aspects to a general architecture associated to any number of quadrotors will be studied. More than three quadrotors will offer redundancy to control the platform, enhancing the robustness of the whole system against quadrotor failures and facilitating reconfiguration tasks.

\section{REFERENCES}

[1] Q. Lindsey, D. Mellinger, and V. Kumar, "Construction of Cubic Structures with Quadrotor Teams," Robotics: Science and Systems, 2011.

[2] S. Kim, S. Choi, and H. J. Kim, "Aerial manipulation using a quadrotor with a two DOF robotic arm," in IEEE International Conference on Intelligent Robots and Systems, Tokyo, Japan, nov 2013, pp. 49904995.

[3] H. Yang and D. Lee, "Dynamics and Control of Quadrotor with Robotic Manipulator," in International Conference on Robotics and Automation, Hong Kong, China, may 2014, pp. 5544-5549.

[4] T. W. Danko, K. P. Chaney, and P. Y. Oh, "A parallel manipulator for mobile manipulating UAVs," in IEEE Conference on Technologies for Practical Robot Applications, Woburn, MA, USA, aug 2015, pp. 1-6.

[5] M. Kamel, K. Alexis, and R. Siegwart, "Design and modeling of dexterous aerial manipulator," in IEEE International Conference on Intelligent Robots and Systems, Daejeon, Korea, oct 2016, pp. 48704876.

[6] K. Sreenath and V. Kumar, "Dynamics, Control and Planning for Cooperative Manipulation of Payloads Suspended by Cables from Multiple Quadrotor Robots," in Robotics: Science and Systems, Berlin, Germany, may 2013.

[7] D. Six, A. Chriette, S. Briot, and P. Martinet, "Dynamic Modeling and Trajectory Tracking Controller of a Novel Flying Parallel Robot," in IFAC World Congress, Toulouse, France, jul 2017.

[8] J. Diebel, "Representing Attitude: Euler Angles, Unit Quaternions, and Rotation Vectors," Standford University, Tech. Rep., 2006.

[9] R. Mahony, V. Kumar, and P. Corke, "Multirotor Aerial Vehicles: Modeling, Estimation, and Control of Quadrotor," IEEE Robotics and Automation Magazine, vol. 19, no. 3, pp. 20-32, 2012.

[10] S. R. S. Ball, A treatise on the theory of screws. Cambridge, UK: Cambridge, University Press, 1900.

[11] K. Hunt, Kinematic Geometry of Mechanisms. Oxford, UK: Oxford University Press, 1978.

[12] V. Kumar, "Instantaneous kinematics of parallel-chain robotic mechanisms," Journal of Mechanical Design, vol. 114, pp. 349-358, 1992.

[13] J. Merlet, Parallel robots. Dordrecht, The Netherland: Springer, 2006.

[14] P. Ben-Horin and M. Shoham, "Application of GrassmannCayley Algebra to Geometrical Interpretation of Parallel Robot Singularities," The International Journal of Robotics Research, vol. 28, no. 1, pp. 127-141, 2009.

[15] _ - "Singularity condition of six-degree-of-freedom three-legged parallel robots based on Grassmann - Cayley Algebra," IEEE Transactions on Robotics, vol. 22, no. 4, pp. 577-590, 2006.

[16] D. Six, S. Briot, A. Chriette, and P. Martinet, "A Controller Avoiding Dynamic Model Degeneracy of Parallel Robots During Singularity Crossing," Journal of Mechanisms and Robotics, vol. 9, no. 5, pp. 051 008-051 008-8, 2017.

[17] J.-P. Merlet, "Singular Configurations of Parallel Manipulators and Grassmann Geometry," The International Journal of Robotics Research, vol. 8, no. 5, pp. 45-56, 1989.

[18] W. Khalil and E. Dombre, Modeling, Identification and Control of Robots. Kogan Page Science, 2002.

[19] H. Voos, "Nonlinear control of a quadrotor micro-uav using feedbacklinearization," in IEEE 2009 International Conference on Mechatronics, no. Apr, Malaga, Spain, 2009.

[20] R. Xu and U. Ozguner, "Sliding Mode Control of a Quadrotor Helicopter," in Proceedings of IEEE Conference on Decision and Control, San Diego, CA, USA, 2006, pp. 4957-4962. 